\title{
Evaluation of Anti-Inflammatory Potential of the New Ganghwaljetongyeum on Adjuvant-Induced Inflammatory Arthritis in Rats
}

\author{
Wangin Kim, ${ }^{1}$ Sangbin Park, ${ }^{1}$ Chanhun Choi, ${ }^{1}$ Youg Ran Kim, ${ }^{2}$ Inkyu Park, ${ }^{3}$ \\ Changseob Seo, ${ }^{4}$ Daehwan Youn, ${ }^{1}$ Wook Shin, ${ }^{1}$ Yumi Lee, ${ }^{1}$ Donghee Choi, ${ }^{1}$ Mirae Kim, \\ Hyunju Lee, ${ }^{5}$ Seonjong Kim, ${ }^{1}$ and Changsu $\mathrm{Na}^{1}$ \\ ${ }^{1}$ College of Korean Medicine, Dongshin University, 185 Geonjae-ro, Naju-si, Jeollanam-do 58245, Republic of Korea \\ ${ }^{2}$ College of Pharmacy, Chonnam National University, 77 Yongbong-ro, Buk-gu, Gwangju 61186, Republic of Korea \\ ${ }^{3}$ College of Medicine, Chonnam National University, 77 Yongbong-ro, Buk-gu, Gwangju 61186, Republic of Korea \\ ${ }^{4}$ Mibyeong Research Center, Korea Institute of Oriental Medicine, 1672 Yuseong-daero, Yuseong-gu, Daejeon 34054, Republic of Korea \\ ${ }^{5}$ School of Information and Communications, Gwangju Institute of Science and Technology, 123 Cheomdangwagi-ro, Buk-gu, \\ Gwangju 61005, Republic of Korea
}

Correspondence should be addressed to Changsu Na; nakugi@hanmail.net

Received 22 October 2015; Revised 22 April 2016; Accepted 24 April 2016

Academic Editor: Ke Ren

Copyright (C) 2016 Wangin Kim et al. This is an open access article distributed under the Creative Commons Attribution License, which permits unrestricted use, distribution, and reproduction in any medium, provided the original work is properly cited.

\begin{abstract}
Ganghwaljetongyeum (GHJTY) has been used as a standard treatment for arthritis for approximately 15 years at the Korean Medicine Hospital of Dongshin University. GHJTY is composed of 18 medicinal herbs, of which five primary herbs were selected and named new Ganghwaljetongyeum (N-GHJTY). The purpose of the present study was to observe the effect of N-GHJTY on arthritis and to determine its mechanism of action. After confirming arthritis induction using complete Freund's adjuvant (CFA) in rats, N-GHJTY $(62.5,125$, and $250 \mathrm{mg} / \mathrm{kg} /$ day $)$ was administered once a day for 10 days. In order to determine pathological changes, edema of the paws and weight were measured before and for 10 days after N-GHJTY administration. Cytokine (TNF- $\alpha$, IL- $1 \beta$, and IL-6) levels and histopathological lesions in the knee joint were also examined. Edema in the paw and knee joint of N-GHJTYtreated rats was significantly decreased at 6,8 , and 10 days after administration, compared to that in the CFA-control group, while weight consistently increased. Rats in N-GHJTY-treated groups also recovered from the CFA-induced pathological changes and showed a significant decline in cytokine levels. Taken together, our results showed that N-GHJTY administration was effective in inhibiting CFA-induced arthritis via anti-inflammatory effects while promoting cartilage recovery by controlling cytokine levels.
\end{abstract}

\section{Introduction}

Arthritis collectively refers to more than 100 rheumatic diseases that are characterized by inflammation, pain, and stiffness in the musculoskeletal system and that range from localized, self-limiting conditions to systemic, autoimmune processes [1]. Arthritis occurs in all age groups and peaks between the ages of 35 and 50 , affecting $~ 1 \%$ of the world's population $[1,2]$. Rheumatoid arthritis (RA) is a systemic inflammatory disease that attacks the joints by producing proliferative synovitis, leading to destruction of articular cartilage and underlying bone. RA affects approximately $0.5 \%$ to $1 \%$ adults worldwide, with women being affected two to three times more frequently than men; RA is also associated with a high mortality rate $[1,3]$.

Autoimmunity and chronic inflammation are activated by an imbalance between pro- and anti-inflammatory cytokines, thereby causing joint damage in RA [4]. RA is characterized by angiogenesis in the synovial membrane, which contributes to the advancement of the disease, as well as the production of inflammatory cells that infiltrate and destroy the synovial tissue $[1,5,6]$. In RA, production of both cytokines and chemokines is induced by macrophage- and fibroblast-derived cytokines [7]. Of these, proinflammatory 
interleukin 1 beta (IL-1 $\beta$ ) and tumor necrosis factor alpha (TNF- $\alpha$ ) are often targeted in RA treatment strategies $[8,9]$.

Our previous study showed that Ganghwaljetongyeum (GHJTY) can effectively attenuate RA by inhibiting synovial cell proliferation as well as the production of proinflammatory mediators from macrophage-like cells [10]. However, 18 medicinal herbs are included in GHJTY, making it impractical for clinical application. In order to improve the efficacy and convenience of pharmaceutically prescribing GHJTY, we used bioinformatics (http://combio.gist.ac.kr/herding) [11] to select the five medicinal herbs with the greatest potential to treat arthritis. The selected medicinal herbs were Ostericum koreanum Maximowicz (Osterici Radix, OK), Lonicera japonica Thunberg (Lonicerae Folium, LJ), Clematis mandshurica Ruprecht (Clematis Radix, CM), Angelica gigas Nakai (Angelicae Gigantis Radix, AG), and Phellodendron amurense Ruprecht (Phellodendri Cortex, PA).

A review of the literature revealed that each of the selected herbs is effective in targeting certain aspects of RA pathophysiology. For example, OK inhibits the production of inflammatory mediators by downregulating nuclear factor kappa beta $(\mathrm{NF}-\kappa \mathrm{B})$ and mitogen-activated protein kinase (MAPK) activity in lipopolysaccharide-stimulated RAW264.7 cells [12]. Additionally, anti-inflammatory activity of the major constituents of LJ has been shown [13], along with the ability of CM extract to interact with NF- $\kappa \mathrm{B}, \mathrm{TNF}-\alpha$, and cyclooxygenase 2 (COX-2) in rats with collagen-induced arthritis [14]. Studies have also shown that AG inhibits focal and systemic inflammation in dinitrofluorobenzene-induced inflammation models and that PA protects against human osteoarthritis by regulating aggrecanases, matrix metalloproteinases (MMPs)/tissue inhibitors of metalloproteinases (TIMP), proinflammatory cytokines, and MAPK pathway signaling $[15,16]$. However, the effects of combining these herbal medicines remain unclear.

The purpose of the present study was to evaluate the efficacy and mechanism of action of this new GHJTY (NGHJTY) using an animal model of RA. Specifically, we used rats with complete Freund's adjuvant- (CFA-) induced arthritis as an experimental animal model used to mimic human $\mathrm{RA}$, as it produces profound systemic inflammation that results in severe joint swelling and remodeling [17]. Moreover, CFA is typically used to investigate therapeutic agents with antiarthritic potential [18]. Our specific aims were to (1) confirm the quality assessment of marker components in $\mathrm{N}$ GHJTY, (2) determine the antiarthritic effects of N-GHJTY by measuring paw edema and observing gross lesions of the paw and knee joint, (3) evaluate potential adverse effects of N-GHJTY by histopathological investigation, and (4) assess the effect of N-GHJTY on proinflammatory cytokines by examining levels of TNF- $\alpha$, IL- $1 \beta$, and IL- 6 .

\section{Materials and Methods}

2.1. Animals. Adult male Sprague-Dawley rats, weighing 200-210 g, were housed in a room with constant temperature $\left(24-26^{\circ} \mathrm{C}\right)$ and humidity (40-60\%). Food (Pellet, GMO, Korea) and water were available ad libitum. Animals were acclimated to the laboratory environment for 1 week before the experiment, and all procedures were approved by the Institutional Animal Care and Use Committee of the Dongshin University (2014-03-02).

2.2. CFA-Induced Arthritis and Drug Administration. In the primary adjuvant-induced arthritis model, $0.25 \mathrm{~mL}$ of CFA (Sigma, St. Louis, MD, USA) was injected into the left hind knee joint. After 7 days, secondary arthritis was induced by injecting $0.05 \mathrm{~mL}$ of CFA under the left hind knee joint and left hind sole. Animals were then divided into the following five groups ( $n=6$ /group): normal, CFA arthritis (CFA-control) and CFA arthritis treated with 62.5, 125, and $250 \mathrm{mg} / \mathrm{kg}$ of N-GHJTY extract per day (N-GHJTY-62.5, NGHJTY-125, and N-GHJTY-250, resp.). Oral administration of N-GHJTY was initiated on the 10th day after arthritis induction and continued for 10 days thereafter. Animals were anesthetized using $2.5 \%$ isoflurane and volumes of the hind paw and knee joint were measured using a Digital Plethysmometer (LE7500, Panlab, Spain) 0, 2, 4, 6, 8, and 10 days after oral N-GHJTY administration.

2.3. Preparation of Herbal Materials. The five herbal medicines forming N-GHJTY were purchased from Omniherb Co. (Yeongcheon, Korea), and their origin was taxonomically confirmed by Professor Jong-Kil Jeong in the Department of Herbology at the College of Oriental Medicine, Dongshin University.

The five herbs (OK, LJ, AG, CM, and PA) were combined in a $6: 4: 4: 4: 3$ ratio. N-GHJTY was prepared via water extraction at $100^{\circ} \mathrm{C}$ for $3 \mathrm{~h}$ and concentrated using a rotary vacuum evaporator (EYELA, Japan) after filtration followed by lyophilization using a vacuum freeze drier (Samwon Freezing Engineering Co., Korea). The yield was about $29.5 \%$.

2.4. Reagents and High-Performance Liquid Chromatography (HPLC) Analysis. Chlorogenic acid (1) and berberine chloride (2) were purchased from Acros Organics (Pittsburgh, PA, USA) and Sigma-Aldrich Co. LLC. (St. Louis, MO, USA), respectively. Nodakenin (3), decursin (6), and decursinol angelate (7) were purchased form NPC BioTechnology (Yeongi, Korea). Isoferulic acid (4) and oxypeucedanin hydrate (5) were purchased from ChemFaces Biochemical Co. Ltd. (Wuhan, China). The purities of the seven reference compounds were $\geq 98.0 \%$ by HPLC analysis and the chemical structures of the seven marker compounds are shown in Figure 1. HPLC-grade solvents, methanol, acetonitrile, and water were obtained from J.T.Baker (Phillipsburg, NJ, USA). Analytical grade formic acid was purchased from SigmaAldrich (St. Louis, MO, USA).

For quality assessment of the seven marker compounds in N-GHJTY, all experiments were conducted using a Shimadzu Prominence LC-20A Series (Shimadzu, Kyoto, Japan) equipped with a solvent delivery unit (LC-20AT), online degasser (DGU-20A3), column oven (CTO-20A), autosample injector (SIL-20AC), and photodiode array (PDA) detector (SPD-M20A). Lab solution software (version 5.54 SP3, Kyoto, Japan) was used for data acquisition and processing. 
<smiles>O=C(/C=C/c1ccc(O)c(O)c1)OC1CC[C@@]2(O)C[C@H](O)[C@H](O)[C@H]1C2</smiles>

Chlorogenic acid (1)<smiles>CC(C)(O[C@@H]1Cc2cc3ccc(=O)oc3cc2O1)[C@H]1O[C@H](CO)[C@@H](O)[C@H](O)[C@H]1O</smiles>

Nodakenin (3)<smiles>COc1ccc2cc3[n+](cc2c1OC)CCc1cc2c(cc1-3)OCO2</smiles>

Berberine chloride (2)<smiles>CC(C)(O)[C@@H](O)COc1c2ccoc2cc2oc(=O)ccc12</smiles>

Oxypeucedanin hydrate (5)<smiles>COc1ccc(/C=C/C(=O)O)cc1O</smiles>

Isoferulic acid (4)<smiles>CC(C)=CC(=O)O[C@H]1Cc2cc3ccc(=O)oc3cc2OC1(C)C</smiles>

Decursin $(6)$<smiles>C/C=C(/C)C(=O)O[C@H]1Cc2cc3ccc(=O)oc3cc2OC1(C)C</smiles>

Decursinol angelate (7)

FIGURE 1: Chemical structure of the seven marker compounds.

Chromatographic separation of all analytes was performed using a Waters SunFire C18 column $(4.6 \times 250 \mathrm{~mm} ; 5 \mu \mathrm{m}$, Milford, MA, USA). The mobile phases consisted of $0.1 \%$ $(\mathrm{v} / \mathrm{v})$ formic acid in distilled water $(\mathrm{A})$ and $0.1 \%(\mathrm{v} / \mathrm{v})$ formic acid in acetonitrile (B) and the gradation condition was optimized as follows: with range of 0-30 min, 10-100\% B; 30$40 \mathrm{~min}, 100 \% \mathrm{~B} ; 40-50 \mathrm{~min}, 100-10 \% \mathrm{~B} ; 50-60 \mathrm{~min}, 10 \% \mathrm{~B}$. The flow rate of mobile phase was maintained at $1.0 \mathrm{~mL} / \mathrm{min}$, and the injection volume was $10 \mathrm{~mL}$. The flow rate was maintained at $1.0 \mathrm{~mL} / \mathrm{min}$, and the injection volume was $10 \mathrm{~mL}$. For HPLC analysis, lyophilized N-GHJTY (200 mg) was dissolved in $20 \mathrm{~mL}$ of $70 \%$ methanol and extracted for $60 \mathrm{~min}$ by sonication. The N-GHJTY extract solution was passed through a $0.2-\mu \mathrm{m}$ syringe filter (PALL Life Sciences, Ann Arbor, MI, USA) before HPLC analysis.

2.5. Blood and Serum Tests. Blood samples were collected, and $100 \mu \mathrm{L}$ was used for a complete blood count (CBC) analysis via a Multispecies Hematology Analyzer (950, Hemavet, USA). Serum was separated from the rest of the blood using a high-speed centrifuge (VS-600CFi, Korea) at $3500 \mathrm{rpm}$
( $g=27.391)$ for $20 \mathrm{~min}$, and aspartate aminotransferase (AST) and alanine transaminase (ALT) levels were measured.

2.6. Measurement of TNF- $\alpha, I L-1 \beta$, and $I L-6$. TNF- $\alpha$ was measured using a Rat TNF- $\alpha$ kit (Invitrogen, USA), IL- $1 \beta$ was assessed using a Rat IL- $1 \beta$ kit (R\&D Systems, USA), and IL-6 was evaluated using a Rat IL-6 kit (Invitrogen, USA). Optical densities (OD) of all samples were measured at $450 \mathrm{~nm}$ via Spectramax (M2, Molecular Devices, USA).

2.7. Hematoxylin and Eosin (HE) Staining. The right knee joint was removed and fixed in Bouin solution for over $24 \mathrm{~h}$. Decalcification was conducted in a $2.5 \%$ nitric acid solution, which was changed once a day for 7 days. The removed tissue was dehydrated using Tissue Processor (Tissue-Tex II, Japan), deparaffinized, stained with HE (Muto, Japan), and observed under an optical microscope (Nikon, Japan).

2.8. Safranin O-Fast Stain. After deparaffinization, the right knee joint was reacted with Weigert's Iron Hematoxylin 
TABLE 1: Changes in paw swelling after N-GHJTY administration in rats with CFA-induced arthritis (mL).

\begin{tabular}{|c|c|c|c|c|c|c|c|}
\hline \multirow{2}{*}{ Group } & \multirow{2}{*}{ Before } & \multicolumn{6}{|c|}{ Days after CFA (days N-GHJTY administration) } \\
\hline & & $10(0)$ & $12(2)$ & $14(4)$ & $16(6)$ & $18(8)$ & $20(10)$ \\
\hline Normal & $1.36 \pm 0.03$ & $1.47 \pm 0.04$ & $1.49 \pm 0.04$ & $1.55 \pm 0.05$ & $1.57 \pm 0.06$ & $1.63 \pm 0.05$ & $1.66 \pm 0.06$ \\
\hline CFA-control & $1.24 \pm 0.01$ & $3.77 \pm 0.25^{\# \#}$ & $3.78 \pm 0.26^{\# \#}$ & $3.91 \pm 0.26^{\# \#}$ & $3.91 \pm 0.28^{\# \#}$ & $3.96 \pm 0.28^{\# \#}$ & $4.00 \pm 0.30^{\# \#}$ \\
\hline N-GHJTY-62.5 & $1.26 \pm 0.03$ & $3.68 \pm 0.33$ & $3.72 \pm 0.31$ & $3.68 \pm 0.26$ & $3.53 \pm 0.23$ & $3.35 \pm 0.21$ & $3.23 \pm 0.17^{*}$ \\
\hline N-GHJTY-125 & $1.24 \pm 0.03$ & $3.68 \pm 0.15$ & $3.67 \pm 0.15$ & $3.60 \pm 0.17$ & $3.36 \pm 0.20$ & $3.15 \pm 0.20^{*}$ & $3.03 \pm 0.20^{*}$ \\
\hline N-GHJTY-250 & $1.31 \pm 0.04$ & $3.63 \pm 0.16$ & $3.66 \pm 0.16$ & $3.49 \pm 0.10$ & $3.22 \pm 0.08^{*}$ & $3.05 \pm 0.05^{*}$ & $2.91 \pm 0.09^{* *}$ \\
\hline
\end{tabular}

Mean \pm SE.

${ }^{\# \#} P<0.01$ versus normal group.

${ }^{* *} P<0.01$ and ${ }^{*} P<0.05$ versus CFA-control group.

(Sigma, USA) solution for $10 \mathrm{~min}$ and stained with $0.001 \%$ Fast Green (Sigma, USA) solution for $5 \mathrm{~min}$. The knee joint tissue was then reacted with $1 \%$ acetate solution for $10 \mathrm{~s}$ and stained with $0.1 \%$ Safranin O (Sigma, USA) solution for $5 \mathrm{~min}$; thereafter, the tissue was dehydrated and observed under an optical microscope (Nikon, Japan).

2.9. Statistical Analysis. Data were analyzed using SPSS 21.0 version for Windows by a nonparametric Mann-Whitney $U$ test. A one-way analysis of variance was conducted on each group, and results are expressed as mean \pm standard error (SE). Comparisons between groups were performed using the post hoc least squared differences (LSD) test. $P<0.05$ and $P<0.01$ were considered statistically significant.

\section{Results}

3.1. Quality Assessment of Seven Marker Components in $\mathrm{N}$-GHJTY. HPLC was performed using the seven marker compounds in N-GHJTY for quality control. The selected compounds were as follows: compound $\mathbf{1}$ (Lonicerae Folium), compound 2 (Phellodendri Cortex), compounds 3, 6, and 7 (Angelicae Gigantis Radix), compound 4 (Clematis Radix), and compound 5 (Osterici Radix). All analytes were separated within $30 \mathrm{~min}$ and the typical three-dimensional chromatogram of the $70 \%$ methanol extract of N-GHJTY is shown in Figure 2. Quantitation was achieved by photodiode array (PDA) detection at $310 \mathrm{~nm}(5), 325 \mathrm{~nm}$ (1 and 4), $330 \mathrm{~nm}$ (6 and 7), $335 \mathrm{~nm}$ (3), and $340 \mathrm{~nm}$ (2) based on retention time and UV spectrum. The retention times of components 1-7 were 8.94, 10.80, 12.00, 12.86, 15.95, 26.02, and $26.24 \mathrm{~min}$, respectively. Using a calibration curve, we determined that correlation coefficients $\left(r^{2}\right)$ of all seven compounds were $\geq 0.9996$. Under optimized chromatography conditions, concentrations of N-GHJTY marker compounds $1-7$ were $5.46 \pm 0.33,1.87 \pm 0.29,1.70 \pm 0.18,1.64 \pm 0.58$, $2.03 \pm 0.33,1.09 \pm 0.02$, and $0.81 \pm 0.01 \mathrm{mg} / \mathrm{g}$, respectively.

\subsection{Effect of N-GHJTY on Gross Lesions of the Paw and Knee} Joint. Paw and knee joint swelling and rubefaction served as external objective indicators for evaluating the severity of the inflammatory arthritic model. The CFA-control group showed rubefaction and hind paw and knee joint swellingboth of which gradually decreased following N-GHJTY

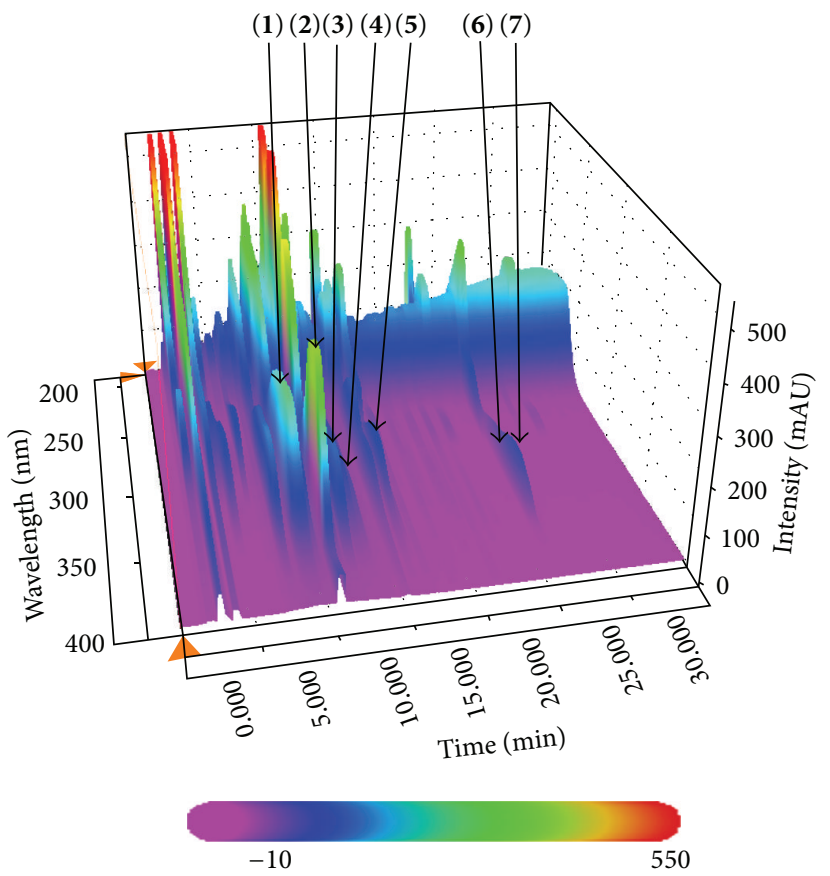

FIgURE 2: Three-dimensional chromatogram of N-GHJTY. Chlorogenic acid (1), berberine chloride (2), nodakenin (3), isoferulic acid (4), oxypeucedanin hydrate (5), decursin (6), and decursinol angelate (7).

treatment at all concentrations $(62.5,125$, and $250 \mathrm{mg} / \mathrm{kg})$ (Figure 3).

3.3. Effect of N-GHJTY on Paw and Knee Joint Swelling. Changes in paw and knee joint swelling are presented in Table 1 . Approximately 3 days after the second immunization, the rat knee joint began to swell and the paw and knee joint were observed to increase in size. On the 10th day, the CFAcontrol group showed a significant increase in both paw and knee joint swellings compared to the normal group. This volume significantly decreased in the N-GHJTY-250 group on the 6th day, the N-GHJTY-125 group on the 8th day, and the N-GHJTY-62.5 group on the 10th day compared to the CFA-control group (Table 1).

3.4. Effect of N-GHJTY on Body Weight. Twelve days after CFA injection, statistically significant reductions in body 


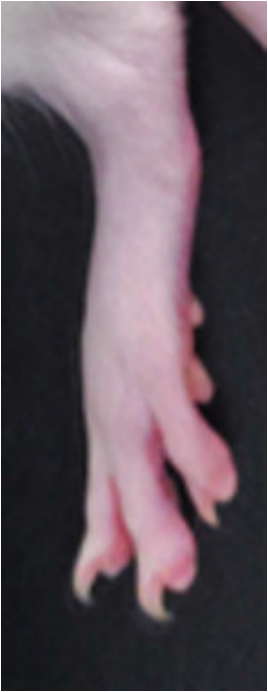

(a)

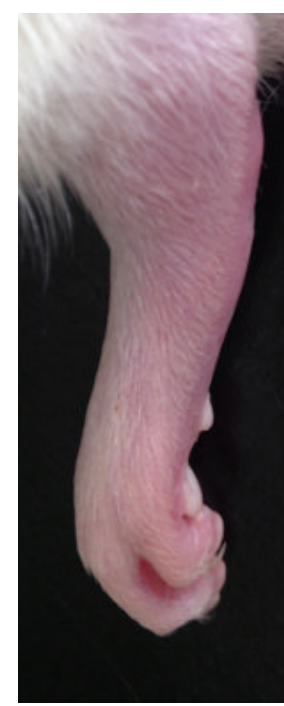

(b)

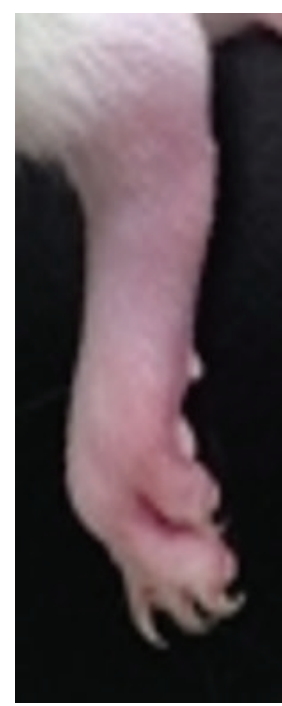

(c)

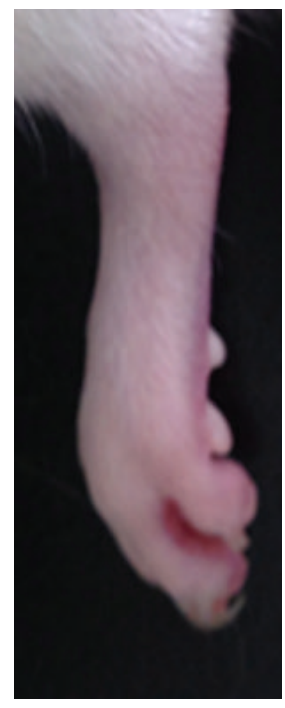

(d)

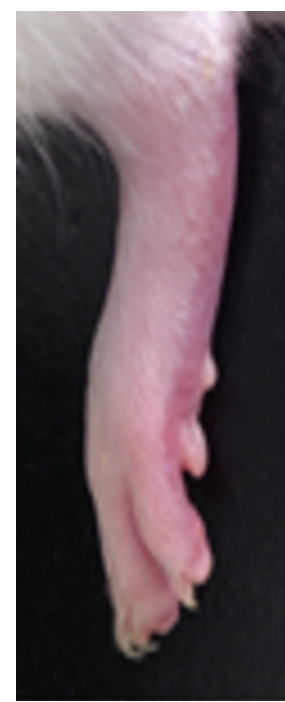

(e)

FIGURE 3: Effect on N-GHJTY in gross lesions in the hind paw and knee swelling in rats with CFA-induced arthritis. (a) Normal group, (b) CFA-control group, (c) N-GHJTY-62.5 group (62.5 mg/kg), (d) N-GHJTY-125 group (125 mg/kg), and (e) N-GHJTY-250 group (250 mg/kg).

TABLE 2: Changes in body weight after N-GHJTY administration in rats with CFA-induced arthritis (\%).

\begin{tabular}{|c|c|c|c|c|c|c|c|}
\hline \multirow{2}{*}{ Group } & \multirow{2}{*}{ Before } & \multicolumn{6}{|c|}{ Days after CFA (days N-GHJTY administration) } \\
\hline & & $10(0)$ & $12(2)$ & $14(4)$ & $16(6)$ & $18(8)$ & $20(10)$ \\
\hline Normal & $100.0 \pm 0.0$ & $145.2 \pm 1.8$ & $148.3 \pm 2.4$ & $153.6 \pm 2.8$ & $159.4 \pm 3.2$ & $163.4 \pm 3.6$ & $164.0 \pm 3.7$ \\
\hline CFA-control & $100.0 \pm 0.0$ & $134.8 \pm 1.7$ & $139.5 \pm 2.2^{\#}$ & $144.7 \pm 3.4$ & $148.9 \pm 4.3$ & $153.4 \pm 5.2$ & $154.0 \pm 5.1$ \\
\hline N-GHJTY-62.5 & $100.0 \pm 0.0$ & $135.5 \pm 2.2$ & $142.9 \pm 1.8$ & $149.6 \pm 1.9$ & $157.4 \pm 2.2$ & $161.4 \pm 1.6$ & $162.1 \pm 1.7$ \\
\hline N-GHJTY-125 & $100.0 \pm 0.0$ & $136.0 \pm 2.5$ & $142.0 \pm 2.3$ & $148.6 \pm 2.5$ & $156.1 \pm 3.2$ & $159.9 \pm 3.6$ & $161.1 \pm 3.6$ \\
\hline N-GHJTY-250 & $100.0 \pm 0.0$ & $136.4 \pm 3.5$ & $143.2 \pm 3.7$ & $149.5 \pm 4.1$ & $157.6 \pm 4.8$ & $161.5 \pm 5.1$ & $162.8 \pm 5.4$ \\
\hline
\end{tabular}

Mean \pm SE.

${ }^{\#} P<0.05$ versus normal group.

TABLE 3: Effect of N-GHJTY on aspartate and alanine aminotransferase levels in rats with CFA-induced arthritis.

\begin{tabular}{lcc}
\hline Group & $\begin{array}{c}\text { Aspartate } \\
\text { aminotransferase }(\mathrm{U} / \mathrm{L})\end{array}$ & $\begin{array}{c}\text { Alanine } \\
\text { aminotransferase }(\mathrm{U} / \mathrm{L})\end{array}$ \\
\hline Normal & $82.0 \pm 5.0$ & $28.4 \pm 1.6$ \\
CFA-control & $93.7 \pm 4.6^{\#}$ & $38.2 \pm 2.9^{\#}$ \\
N-GHJTY-62.5 & $86.0 \pm 7.8$ & $39.0 \pm 2.1$ \\
N-GHJTY-125 & $85.4 \pm 1.9$ & $28.7 \pm 3.4$ \\
N-GHJTY-250 & $80.0 \pm 3.0^{*}$ & $28.8 \pm 1.3^{*}$
\end{tabular}

Mean \pm SE.

${ }^{\#} P<0.05$ versus normal group.

${ }^{*} P<0.05$ versus CFA-control group.

weight were observed in the CFA-control group when compared to the normal group. N-GHJTY treatment at all concentrations $(62.5,125$, and $250 \mathrm{mg} / \mathrm{kg})$ resulted in an increase in body weight; however, this change was not significant (Table 2).

3.5. Effect of N-GHJTY on Transaminase Levels. Aspartate aminotransferase (AST) and alanine aminotransferase (ALT) levels are indicated in Table 3. As shown in the results, only
TABLE 4: Effect of N-GHJTY on TNF- $\alpha$, IL-1 $\beta$, and IL-6 levels in rats with CFA-induced arthritis.

\begin{tabular}{lccc}
\hline Group & TNF- $\alpha(\mathrm{pg} / \mathrm{mL})$ & IL-1 $\beta(\mathrm{pg} / \mathrm{mL})$ & IL-6 $(\mathrm{pg} / \mathrm{mL})$ \\
\hline Normal & $2.33 \pm 0.12$ & $22.9 \pm 0.95$ & $4.3 \pm 0.41$ \\
CFA-control & $4.85 \pm 0.67^{\# \#}$ & $36.7 \pm 0.94^{\# \#}$ & $18.0 \pm 0.87^{\# \#}$ \\
N-GHJTY-62.5 & $3.24 \pm 0.32^{*}$ & $26.8 \pm 0.84^{*}$ & $11.0 \pm 0.5^{*}$ \\
N-GHJTY-125 & $3.01 \pm 0.18^{*}$ & $26.2 \pm 0.52^{* *}$ & $10.4 \pm 0.6^{*}$ \\
N-GHJTY-250 & $2.84 \pm 0.17^{*}$ & $25.4 \pm 0.84^{* *}$ & $9.7 \pm 0.39^{*}$ \\
\hline
\end{tabular}

Mean \pm SE.

${ }^{\# \#} P<0.01$ versus normal group.

${ }^{* *} P<0.01$ and ${ }^{*} P<0.05$ versus CFA-control group.

the N-GHJTY-250 group showed a significant decrease in AST and ALT when compared to CFA-control group rats.

3.6. Effect of N-GHJTY on Proinflammatory Cytokines. TNF$\alpha$, IL- $1 \beta$, and IL- 6 levels are indicated in Table 4 . As shown in the results, TNF- $\alpha$, IL- $1 \beta$, and IL- 6 in the CFA-control group showed a significant increase when compared to levels in the normal group rats. A significant decrease in TNF- $\alpha$, IL$1 \beta$, and IL- 6 levels was observed in all N-GHJTY treatment 


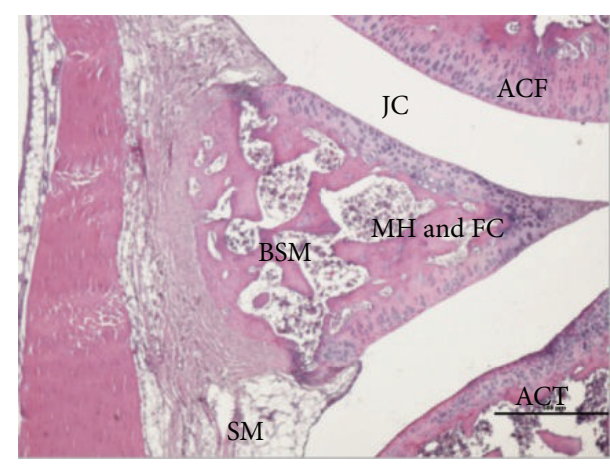

(a)

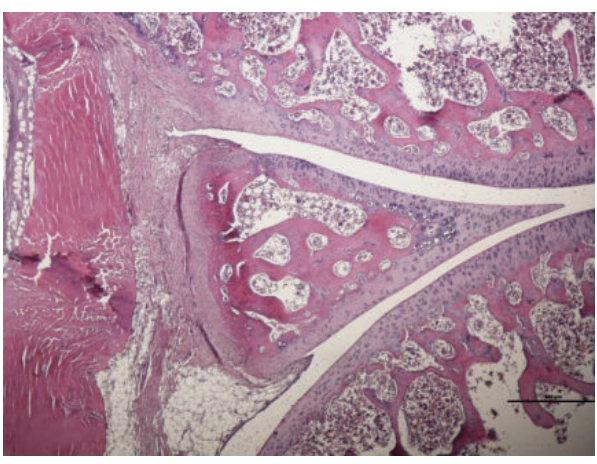

(c)

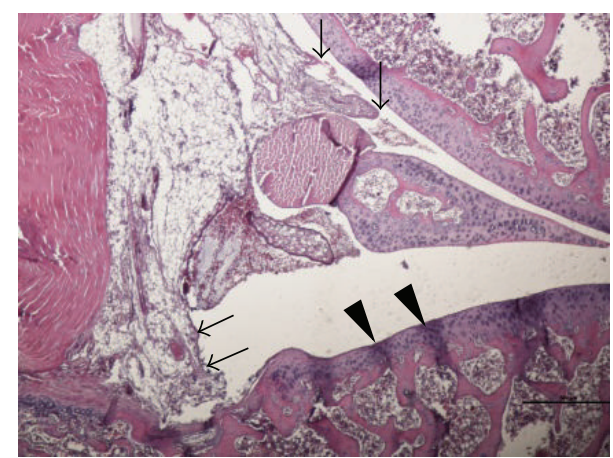

(b)

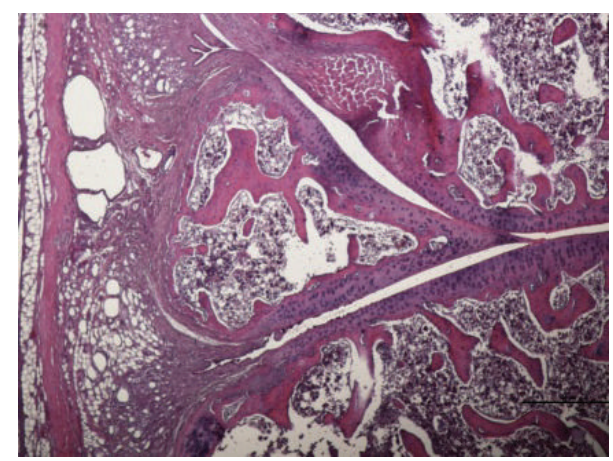

(d)

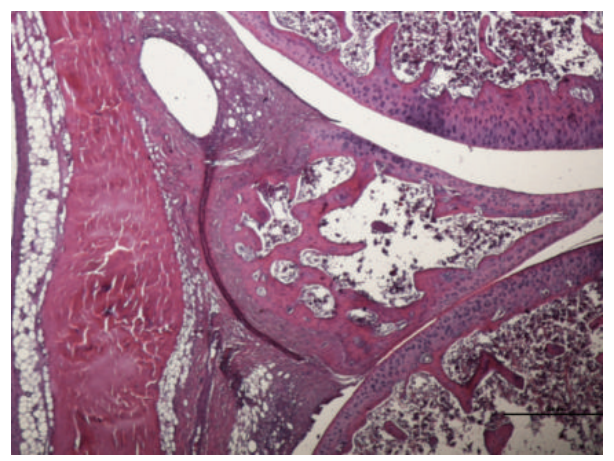

(e)

FIGURE 4: Influence of N-GHJTY on CFA-induced histopathological changes in the knee joints of CFA-induced arthritic rats. Arrows ( $\downarrow$ ) indicate a damaged synovial membrane. Arrow heads $(\boldsymbol{\nabla})$ indicate compressed articular cartilage in the CFA control. (a) Normal group, (b) CFA-control group, (c) N-GHJTY-62.5 group (62.5 mg/kg), (d) N-GHJTY-125 group (125 mg/kg), and (e) N-GHJTY-250 group (250 mg/kg). JC: joint cavity. ACF: articular cartilage of the femur. ACT: articular cartilage of the tibia. BSM: bony spicule within the meniscus. SM: synovial membrane. MH\&FC: meniscus of hyaline and fibrocartilage. HE stain, scale bars $=500 \mathrm{nd}$.

groups $(62.5,125$, and $250 \mathrm{mg} / \mathrm{kg})$ when compared to the CFA-control group rats (Table 4 ).

\subsection{Effects of N-GHJTY on Histopathological Changes Assessed} with HE Staining. Representative HE stained histopathological lesions in the hind knee joint of normal, CFAcontrol, N-GHJTY-62.5, N-GHJTY-125, and N-GHJTY-250 groups are shown in Figure 4. Loose synovial membrane, membrane destruction, disorganized cell arrangement, and compressed cartilage were observed in the CFA-control group (Figure 4(b)). Histopathological changes improved in all N-GHJTY groups $(62.5,125$, and $250 \mathrm{mg} / \mathrm{kg})$ compared to the CFA-control group. These groups presented close synovial membrane and regular cartilage; the surface of the cartilage in the tibia and femur was smooth and exhibited no noticeable damage (Figures 4(c), 4(d), and 4(e)).

\subsection{Effects of N-GHJTY on Histopathological Changes Assessed} with Safranin O-Fast Staining. Safranin O-fast stain was conducted to observe histopathological changes in the knee joint. In the normal group (Figure 5(a)), a positive reaction to proteoglycans in the calcified zone was observed and the cartilage was even. The CFA-control group exhibited little positive reactions, and chondrocyte nuclei appeared 


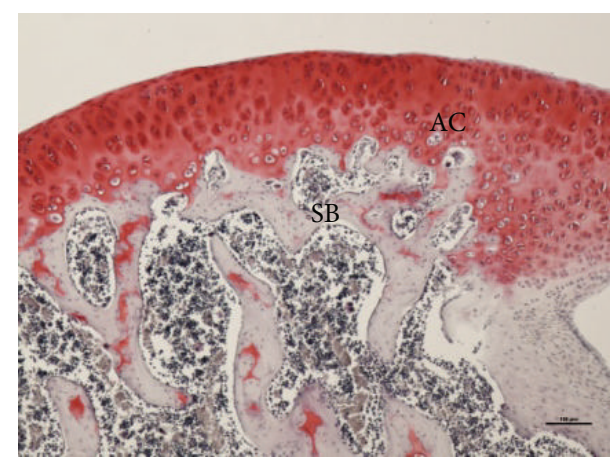

(a)

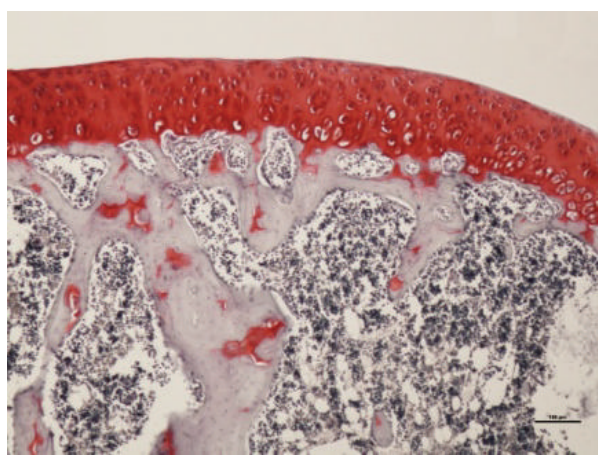

(c)

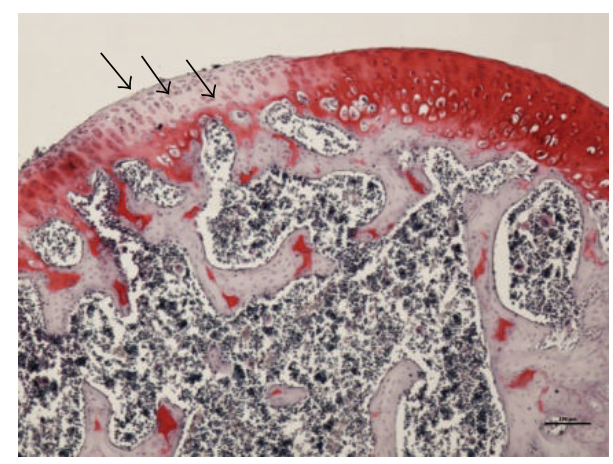

(b)

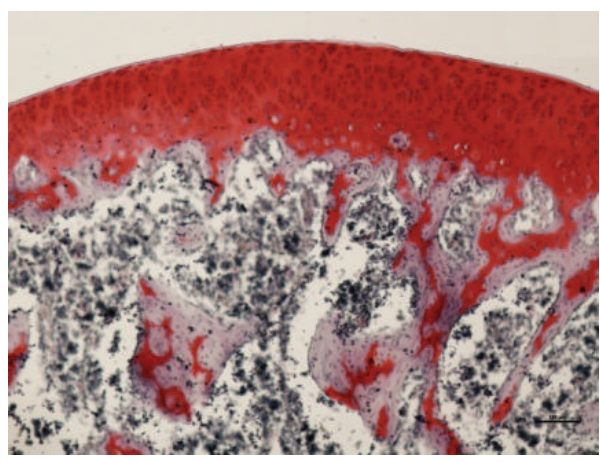

(d)

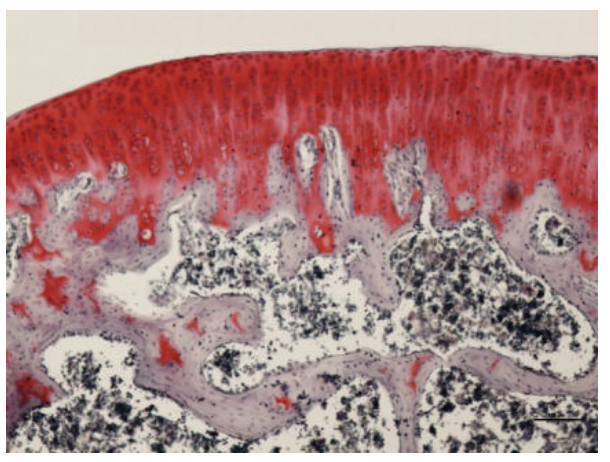

(e)

Figure 5: Influence of N-GHJTY on CFA-induced histopathological changes in the knee joints of CFA-induced arthritic rats. A number of shrunken nuclei (arrows $\downarrow$ ) were observed in the CFA control. (a) Normal group, (b) CFA-control group, (c) N-GHJTY-62.5 group $(62.5 \mathrm{mg} / \mathrm{kg})$, (d) N-GHJTY-125 group (125 mg/kg), and (e) N-GHJTY-250 group (250 mg/kg). AC: articular cartilage. SB: spongy bone. Safranin O-fast stain, scale bars $=100 \mu \mathrm{m}$.

contracted when compared to nuclei in the normal group (Figure 5(b)). N-GHJTY-62.5, N-GHJTY-125, and NGHJTY-250 groups exhibited a greater number of positive reactions in the calcified zone of the cartilage when compared to the CFA-control group (Figures 5(c), 5(d), and 5(e)).

\section{Discussion}

RA is an abnormal autoimmune disease that causes synovial inflammation and damage to joint structure. One characteristic that is specific to RA is the network of new blood vessels that extensively develops in the synovial membrane. This destructive vascular tissue, which is called pannus, extends from the synovium to invade the junction between the cartilage and subchondral bone. With progression of the disease, joint inflammation and the resulting structural changes caused by pannus lead to reduced joint motion, possible ankyloses, joint instability, muscle atrophy from disuse, stretching of the ligaments, and involvement of the tendons and muscles [1].

The CFA approach developed by Pearson [19] is a widely used arthritic model, which is induced in susceptible strains of rats via injection of heat-killed mycobacterium tuberculosis [20]. After CFA injection, a rapid, reliable, robust, and easily measurable polyarthritis develops [21]. Importantly, the joint pathology seen in the rat model shares the synovial 
hyperplasia and cartilage degradation observed in human arthritis, particularly RA [22-24]. CFA has recently become a popular tool to observe the efficacy of herbal medicines for treating arthritis. For example, Zhang et al. used the CFA approach to demonstrate the antiarthritic effect of an extract from Dioscorea zingiberensis C.H. Wright [25]. Additionally, Zhang et al. used CFA to demonstrate antioxidant effects of Genkwa flos flavonoids [26], while Obiri et al. demonstrated that Xylopia aethiopica (Annonaceae) fruit extract suppresses adjuvant-induced arthritis in rats [27]. Several studies have also shown that herbal medicines are effective in treating RA in humans. Shao Li et al. reported that Qing-Luo-Yin extract may have a protective effect against excessive tissue breakdown, angiogenesis, and degradation of extracellular matrix in RA [28]. Chi Zhang et al. also reported that some herbal medicines have beneficial effects on pain management and swollen joint relief in individuals with RA [29]. Additionally, Liu et al. reported that Xinfeng, a patent Chinese herbal medicine, is effective and safe in the treating RA [30].

In the current study, we found that treatment with the $\mathrm{N}$-GHJTY resulted in a gradual decrease of CFA-induced paw and knee joint swelling, as well as rubefaction. This finding was present in all N-GHJTY-treated groups (62.5, 125 , and $250 \mathrm{mg} / \mathrm{kg}$ ) and exhibited a dose-dependent effectwith the N-GHJTY-250 group showing the greatest decrease in paw swelling (Figure 3). In terms of time course, the $\mathrm{N}$ GHJTY-250 group showed significantly less paw and knee joint swelling on the 6th day after treatment, the N-GHJTY125 group on the 8th day, and the N-GHJTY-62.5 group on the 10th day (Table 1). Moreover, rats in all N-GHJTY groups $(62.5,125$, and $250 \mathrm{mg} / \mathrm{kg})$ exhibited increases in body weight when compared to those in the CFA-control group.

Regarding proinflammatory cytokines, all N-GHJTYtreated groups $(62.5,125$, and $250 \mathrm{mg} / \mathrm{kg})$ displayed significant reductions in TNF- $\alpha$, IL- $1 \beta$, and IL- 6 when compared to rats in the CFA-control group. RA is initiated by a $\mathrm{T}$ cell-mediated immune response that stimulates the release of cytokines and promotes antibody formation, which leads to destruction of the joint [1]. Specifically, TNF- $\alpha$ induces the production of IL- $1 \beta$ and IL- 6 [31], with the former being a crucial mediator of the inflammatory response, causing various autoinflammatory syndromes $[32,33]$, and the latter being secreted by $\mathrm{T}$ cells and macrophages to stimulate the immune response $[34,35]$. Thus, proinflammatory cytokines (particularly IL-1 $\beta$, IL-6, and TNF- $\alpha$ ) play an important role in arthritis onset [36], while inhibitors of these cytokines are effective in controlling chronic inflammation [37, 38].

The protective effects of N-GHJTY were confirmed by our histopathological investigations with HE and Safranin O-fast staining. Specifically, rats in the CFA-control group exhibited loose synovial membrane, membrane destruction, disorganized cell arrangement, and compressed cartilage-all of which were prevented in rats treated with N-GHJTY. As mentioned, release of enzymes and inflammatory mediators from damaged tissues perpetuates the inflammatory process [1]. It has also been shown that cytokines released by inflamed synovial tissue can reach systemic circulation and act on other organs [39]. Rheumatoid factor (RF), which is an autoantibody found in RA, forms an immune complex with immunoglobulin $\mathrm{G}$ that contributes to RA progression by further triggering inflammatory responses and attracting inflammatory cells. Thus, the ability of N-GHJTY to protect against cartilage and synovial membrane destruction could additionally prevent systemic inflammation by preventing damaged synovial tissue from releasing cytokines.

To investigate if repeated N-GHJTY treatment could induce toxicity, we observed transaminase levels (AST and ALT) in all N-GHJTY-treated groups. Our findings revealed that N-GHJTY treatment did not alter either AST or ALT, suggesting that N-GHJTY was not toxic to rats. Moreover, investigations of various diseases (including arthritis) have reported that AST and ALT leak into the blood stream in proportion to the extent of tissue damage [40-42]. Indeed, transaminase levels were slightly increased in the CFAcontrol group in the current study. Consistent with our findings on the anti-inflammatory effects of N-GHJTY, we observed that the highest dose of this new herbal combination (i.e., $250 \mathrm{mg} / \mathrm{kg}$ ) resulted in a slight decrease of transaminase levels, suggesting improved liver function in these animals.

Taken together, our findings suggest that N-GHJTY administration could prevent inflammatory cells from infiltrating and destroying synovial tissue and could also suppress the release of proinflammatory cytokines. Moreover, NGHJTY was effective in preventing the destruction of joint tissue and facilitated the repair of CFA-induced injury to the joint cartilage, resulting in reduced paw and knee swelling. Thus, the present study provides evidence supporting the clinical use of N-GHJTY for treating arthritis.

\section{Conclusions}

N-GHJTY, a new complex herbal medication, was effective in treating a rat model of inflammatory arthritis. Specifically, N-GHJTY significantly suppressed the progression of CFAinduced arthritis, as was evident from the decrease in paw and knee joint swelling, and was effective in preventing articular cartilage and synovial tissue degeneration. We also revealed that the protective mechanisms of N-GHJTY treatment could be partially explained by a decrease in the proinflammatory cytokines, TNF- $\alpha$, IL- $1 \beta$, and IL- 6 . Additional studies are required to determine other molecular mechanisms associated with N-GHJTY administration, as well as specific therapeutic effects.

\section{Competing Interests}

The authors declare no competing interests regarding the publication of this paper.

\section{Authors' Contributions}

Wangin Kim and Sangbin Park contributed equally to this work. 


\section{Acknowledgments}

This study was supported by a Grant (no. HI13C2285) from the Korea Healthcare Technology R\&D Project, Ministry for Health \& Welfare Affairs, Republic of Korea.

\section{References}

[1] C. M. Porth, Essentials of Pathophysiology: Concepts of Altered Health States, Wolters Kluwer Health/Lippincott Williams \& Wilkins, 3rd edition, 2011.

[2] A. J. MacGregor and A. J. Silman, "Rheumatoid arthritis and other synovial disorders: classification and epidemiology," in Rheumatology, M. C. Hochberg, A. J. Silman, J. S. Smolen, M. E. Weinblatt, and M. H. Weisman, Eds., vol. 1, Mosby, London, UK, 2004.

[3] S. E. Gabriel and K. Michaud, "Epidemiological studies in incidence, prevalence, mortality, and comorbidity of the rheumatic diseases," Arthritis Research and Therapy, vol. 11, no. 3, article 229, 2009.

[4] I. B. McInnes and G. Schett, "Cytokines in the pathogenesis of rheumatoid arthritis," Nature Reviews Immunology, vol. 7, no. 6, pp. 429-442, 2007.

[5] G. S. Firestein, "Evolving concepts of rheumatoid arthritis," Nature, vol. 423, no. 6937, pp. 356-361, 2003.

[6] Y. Okada, D. Wu, G. Trynka et al., "Genetics of rheumatoid arthritis contributes to biology and drug discovery," Nature, vol. 506, no. 7488, pp. 376-381, 2014.

[7] D. E. Furst and P. Emery, "Rheumatoid arthritis pathophysiology: update on emerging cytokine and cytokine-associated cell targets," Rheumatology, vol. 53, no. 9, pp. 1560-1569, 2014.

[8] B. Bresnihan, J. M. Alvaro-Gracia, M. Cobby et al., "Treatment of rheumatoid arthritis with recombinant human interleukin-1 receptor antagonist," Arthritis and Rheumatism, vol. 41, no. 12, pp. 2196-2204, 1998.

[9] Y. Zhuang, S. Lyn, Y. Lv et al., "Pharmacokinetics and safety of golimumab in healthy Chinese subjects following a single subcutaneous administration in a randomized phase I trial," Clinical Drug Investigation, vol. 33, no. 11, pp. 795-800, 2013.

[10] B.-R. Jeoung, K. D. Lee, C.-S. Na, Y.-E. Kim, B. Kim, and Y. R. Kim, "Ganghwaljetongyeum, an anti-arthritic remedy, attenuates synoviocyte proliferation and reduces the production of proinflammatory mediators in macrophages: the therapeutic effect of GHJTY on rheumatoid arthritis," BMC Complementary and Alternative Medicine, vol. 13, article 47, 2013.

[11] W. Choi, C. Choi, Y. R. Kim, S. Kim, C. Na, and H. Lee, "HerDing: herb recommendation system to treat diseases using genes and chemicals," Database, vol. 2016, Article ID baw011, 2016.

[12] H. W. Jung, R. Mahesh, J. H. Park, Y. C. Boo, K. M. Park, and Y.K. Park, "Bisabolangelone isolated from Ostericum koreanum inhibits the production of inflammatory mediators by downregulation of NF- $\kappa \mathrm{B}$ and ERK MAP kinase activity in LPSstimulated RAW264.7 cells," International Immunopharmacology, vol. 10, no. 2, pp. 155-162, 2010.

[13] S. J. Lee, E. J. Shin, K. H. Son, H. W. Chang, S. S. Kang, and H. P. Kim, "Anti-inflammatory activity of the major constituents of Lonicera japonica," Archives of Pharmacal Research, vol. 18, no. 2, pp. 133-135, 1995.

[14] C. Peng, P. K. Perera, Y.-M. Li, W.-R. Fang, L.-F. Liu, and F.W. Li, "Anti-inflammatory effects of Clematis chinensis Osbeck
extract(AR-6) may be associated with NF- $\kappa$ B, TNF- $\alpha$, and COX-2 in collagen-induced arthritis in rat," Rheumatology International, vol. 32, no. 10, pp. 3119-3125, 2012.

[15] S. S. Joo, D. S. Park, S. H. Shin et al., "Anti-allergic effects and mechanisms of action of the ethanolic extract of Angelica gigas in dinitrofluorobenzene-induced inflammation models," Environmental Toxicology and Pharmacology, vol. 30, no. 2, pp. 127-133, 2010.

[16] J.-H. Kim, J.-E. Huh, Y.-H. Baek, J.-D. Lee, D.-Y. Choi, and D.-S. Park, "Effect of Phellodendron amurense in protecting human osteoarthritic cartilage and chondrocytes," Journal of Ethnopharmacology, vol. 134, no. 2, pp. 234-242, 2011.

[17] R. Roubenoff, L. M. Freeman, D. E. Smith, L. W. Abad, C. A. Dinarello, and J. J. Kehayias, "Adjuvant arthritis as a model of inflammatory cachexia," Arthritis and Rheumatism, vol. 40, no. 3, pp. 534-539, 1997.

[18] A. Omoto, Y. Kawahito, I. Prudovsky et al., "Copper chelation with tetrathiomolybdate suppresses adjuvant-induced arthritis and inflammation-associated cachexia in rats," Arthritis Research \& Therapy, vol. 7, no. 6, pp. R1174-R1182, 2005.

[19] C. M. Pearson, "Development of arthritis, periarthritis and periostitis in rats given adjuvants," Experimental Biology and Medicine, vol. 91, no. 1, pp. 95-101, 1956.

[20] M. Durai, H. R. Kim, and K. D. Moudgil, "The regulatory Cterminal determinants within mycobacterial heat shock protein 65 are cryptic and cross-reactive with the dominant self homologs: implications for the pathogenesis of autoimmune arthritis," The Journal of Immunology, vol. 173, no. 1, pp. 181-188, 2004.

[21] S. S. Patel and P. V. Shah, "Evaluation of anti-inflammatory potential of the multidrug herbomineral formulation in male Wistar rats against rheumatoid arthritis," Journal of Ayurveda and Integrative Medicine, vol. 4, no. 2, pp. 86-93, 2013.

[22] M. L. Andersen, E. H. R. Santos, M. D. L. V. Seabra, A. A. B. da Silva, and S. Tufik, "Evaluation of acute and chronic treatments with Harpagophytum procumbens on Freund's adjuvantinduced arthritis in rats," Journal of Ethnopharmacology, vol. 91, no. 2-3, pp. 325-330, 2004.

[23] A. Bendele, J. Mccomb, T. Gould et al., "Animal models of arthritis: relevance to human disease," Toxicologic Pathology, vol. 27, no. 1, pp. 134-142, 1999.

[24] J. D. Taurog, D. C. Argentieri, and R. A. McReynolds, "Adjuvant arthritis," Methods in Enzymology, vol. 162, pp. 339-355, 1988.

[25] X.-X. Zhang, Y. Ito, J.-R. Liang, J.-L. Liu, J. He, and W.-J. Sun, "Therapeutic effects of total steroid saponin extracts from the rhizome of Dioscorea zingiberensis C.H.Wright in Freund's complete adjuvant induced arthritis in rats," International Immunopharmacology, vol. 23, no. 2, pp. 407-416, 2014.

[26] C.-F. Zhang, S.-L. Zhang, X. He et al., "Antioxidant effects of Genkwa flos flavonoids on Freund's adjuvant-induced rheumatoid arthritis in rats," Journal of Ethnopharmacology, vol. 153, no. 3, pp. 793-800, 2014.

[27] D. D. Obiri, N. Osafo, P. G. Ayande, and A. O. Antwi, "Xylopia aethiopica (Annonaceae) fruit extract suppresses Freunds adjuvant-induced arthritis in Sprague-Dawley rats," Journal of Ethnopharmacology, vol. 152, no. 3, pp. 522-531, 2014.

[28] S. Li, A.-P. Lu, Y.-Y. Wang, and Y.-D. Li, "Suppressive effects of a Chinese herbal medicine Qing-Luo-Yin extract on the angiogenesis of collagen-induced arthritis in rats," The American Journal of Chinese Medicine, vol. 31, no. 5, pp. 713-720, 2003.

[29] C. Zhang, M. Jiang, and A. Lu, "Chinese herbal medicines versus disease modifying antirheumatic drugs for management 
of rheumatoid arthritis: a systematic review," European Journal of Integrative Medicine, vol. 3, no. 3, pp. e219-e231, 2011.

[30] J. Liu, C.-B. Huang, Y. Wang et al., "Chinese herbal medicine Xinfeng Capsule in treatment of rheumatoid arthritis: study protocol of a multicenter randomized controlled trial," Journal of Integrative Medicine, vol. 11, no. 6, pp. 428-434, 2013.

[31] F. M. Brennan and I. B. McInnes, "Evidence that cytokines play a role in rheumatoid arthritis," Journal of Clinical Investigation, vol. 118, no. 11, pp. 3537-3545, 2008.

[32] RefSeq, "RefSeq: NCBI Reference Sequence Database," 2008, http://www.ncbi.nlm.nih.gov/gene?Db=gene\&Cmd=ShowDetailView\&TermToSearch=3553.

[33] S. L. Masters, A. Simon, I. Aksentijevich, and D. L. Kastner, "Horror autoinflammaticus: the molecular pathophysiology of autoinflammatory disease," Annual Review of Immunology, vol. 27, pp. 621-668, 2009.

[34] T. Van Der Poll, C. V. Keogh, X. Guirao, W. A. Buurman, M. Kopf, and S. F. Lowry, "Interleukin-6 gene-deficient mice show impaired defense against pneumococcal pneumonia," Journal of Infectious Diseases, vol. 176, no. 2, pp. 439-444, 1997.

[35] M. A. Febbraio and B. K. Pedersen, "Contraction-induced myokine production and release: is skeletal muscle an endocrine organ?" Exercise \& Sport Sciences Reviews, vol. 33, no. 3, pp. 114-119, 2005.

[36] E. H. S. Choy and G. S. Panayi, "Cytokine pathways and joint inflamation in rheumatoid arthritis," The New England Journal of Medicine, vol. 344, no. 12, pp. 907-916, 2001.

[37] K. J. Tracey, "The inflammatory reflex," Nature, vol. 420, no. 6917, pp. 853-859, 2002.

[38] V. A. Pavlov and K. J. Tracey, "Controlling inflammation: the cholinergic anti-inflammatory pathway," Biochemical Society Transactions, vol. 34, no. 6, pp. 1037-1040, 2006.

[39] N. Sattar, D. W. McCarey, H. Capell, and I. B. McInnes, "Explaining how 'high-grade' systemic inflammation accelerates vascular risk in rheumatoid arthritis," Circulation, vol. 108, no. 24, pp. 2957-2963, 2003.

[40] E. G. Giannini, R. Testa, and V. Savarino, "Liver enzyme alteration: a guide for clinicians," Canadian Medical Association Journal, vol. 172, no. 3, pp. 367-379, 2005.

[41] C. I. B. Walker, G. Trevisan, M. F. Rossato et al., "Antinociceptive effect of Mirabilis jalapa on acute and chronic pain models in mice," Journal of Ethnopharmacology, vol. 149, no. 3, pp. 685693, 2013.

[42] A. A. Adeneye, A. I. Oreagba, I. O. Ishola, and H. A. Kalejaiye, "Evaluation of the anti-arthritic activity of the hydroethanolic leaf extract of Alchornea cordifolia in rats," African Journal of Traditional, Complementary and Alternative Medicines, vol. 11, no. 2, pp. 402-410, 2014. 


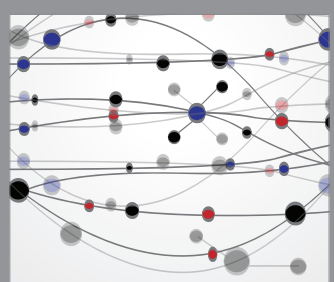

The Scientific World Journal
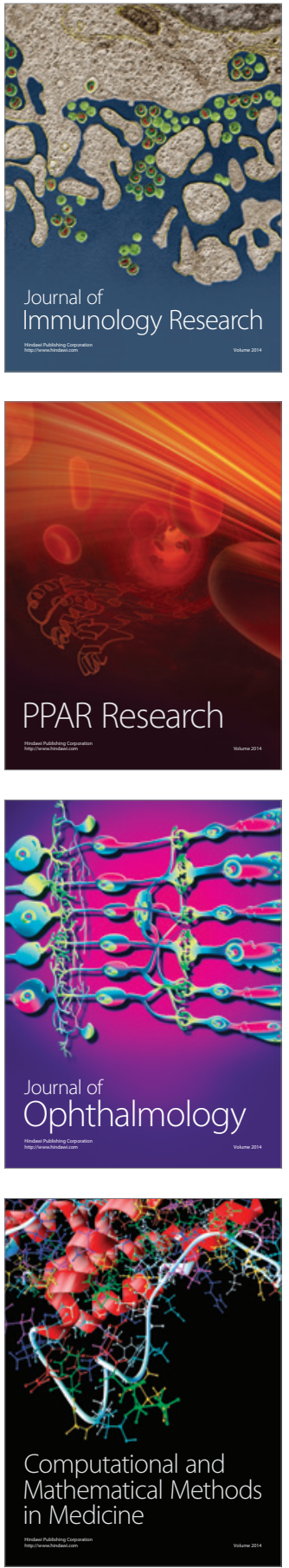

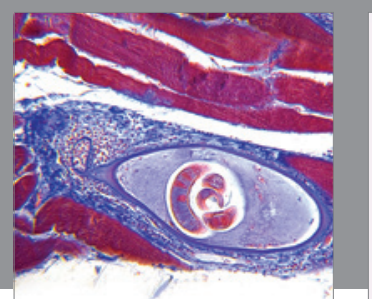

Gastroenterology Research and Practice

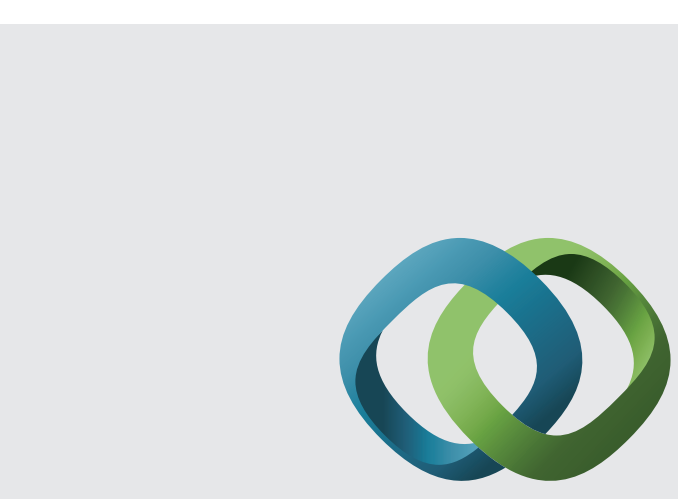

\section{Hindawi}

Submit your manuscripts at

http://www.hindawi.com
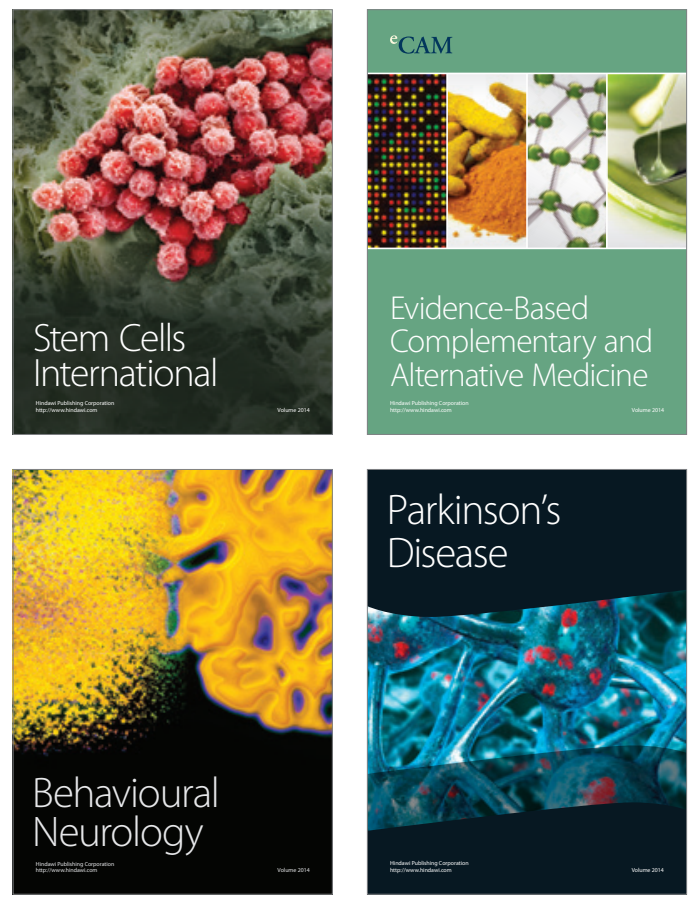
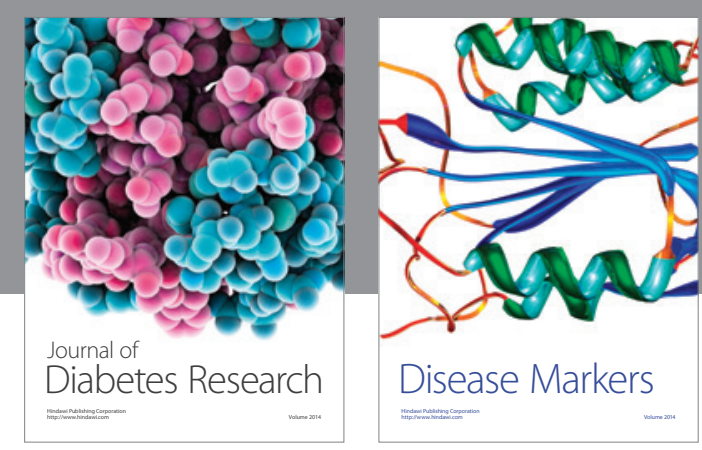

Disease Markers
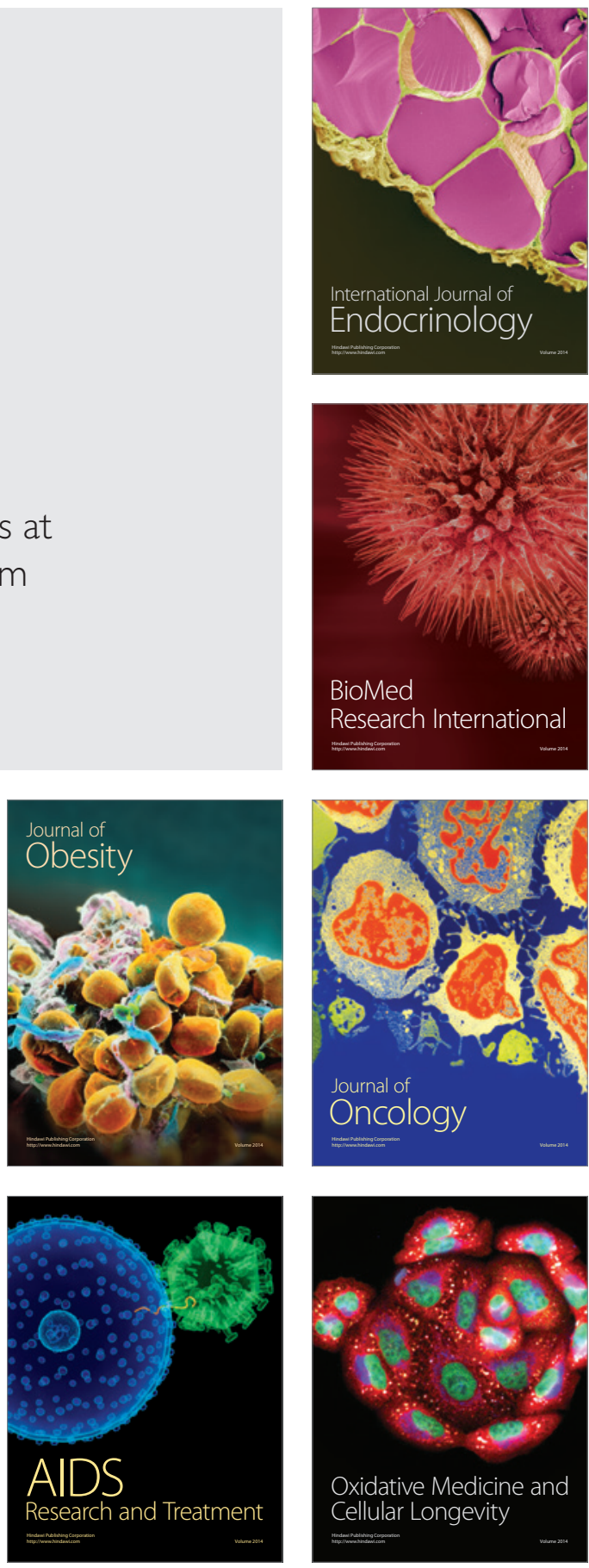